\title{
Correct Implementation of Polarization Constants in Wurtzite Materials and Impact on III-Nitrides
}

\author{
Cyrus E. Dreyer, ${ }^{1,2}$ Anderson Janotti, ${ }^{1,{ }^{*}}$ Chris G. Van de Walle, ${ }^{1}$ and David Vanderbilt ${ }^{2}$ \\ ${ }^{1}$ Materials Department, University of California, Santa Barbara, California 93106-5050, USA \\ ${ }^{2}$ Department of Physics and Astronomy, Rutgers University, Piscataway, New Jersey 08845-0849, USA
}

(Received 22 December 2015; published 20 June 2016)

Accurate values for polarization discontinuities between pyroelectric materials are critical for understanding and designing the electronic properties of heterostructures. For wurtzite materials, the zincblende structure has been used in the literature as a reference to determine the effective spontaneous polarization constants. We show that, because the zincblende structure has a nonzero formal polarization, this method results in a spurious contribution to the spontaneous polarization differences between materials. In addition, we address the correct choice of "improper" versus "proper" piezoelectric constants. For the technologically important III-nitride materials GaN, AlN, and InN, we determine polarization discontinuities using a consistent reference based on the layered hexagonal structure and the correct choice of piezoelectric constants, and discuss the results in light of available experimental data.

DOI: 10.1103/PhysRevX.6.021038

\section{INTRODUCTION}

Pyroelectric materials have emerged in a variety of electronic and optoelectronic applications. Because of the symmetry of their crystal structure, these materials exhibit spontaneous (SP) and piezoelectric (PZ) dipole moments [1], which manifest themselves as electric fields in heterostructure layers and sheet charges at interfaces. In the technologically important III-nitrides, which have the wurtzite (WZ) structure (space group $P 6_{3} m c$ ), polarization differences allow for strong carrier confinement and the formation of a two-dimensional electron gas (2DEG) with high density at $\mathrm{AlGaN} / \mathrm{GaN}$ interfaces, exploited in high electron mobility transistors (HEMTs). The effect of polarization can also be detrimental, for example, causing the quantum-confined Stark effect in quantum wells of light-emitting diodes (LEDs), which reduces radiative recombination rates and shifts the emission wavelength. For both HEMTs and LEDs, accurate values of the SP and PZ polarization constants are required for a fundamental understanding as well as for device design.

Since experimental determination of the separate SP and PZ contributions to the total polarization is very difficult, calculated values of SP and PZ polarization constants are widely used in simulations. The PZ polarization constants are, in principle, fairly straightforward to explicitly measure

\footnotetext{
*Present address: Materials Science and Engineering, University of Delaware, Newark, Delaware 19716-1501, USA.

Published by the American Physical Society under the terms of the Creative Commons Attribution 3.0 License. Further distribution of this work must maintain attribution to the author(s) and the published article's title, journal citation, and DOI.
}

Subject Areas: Condensed Matter Physics, Materials Science, Optoelectronics or calculate [2]. However, the reported values exhibit a considerable spread [3]. In addition, the difference between so-called "proper" and "improper" $\mathrm{PZ}$ constants $[2,4]$ is often overlooked, even though it can give rise to significant quantitative changes in the resulting polarization fields. This difference is one issue that is elucidated in the present paper.

The definition of SP polarization constants is even more subtle, and they are typically not amenable to explicit experimental determination, except in special cases [5]. The calculation of SP polarization requires the choice of a reference structure, which in the case of WZ semiconductors, has invariably been chosen to be zincblende (ZB) [6-8]. In this work, we show that, although $\mathrm{ZB}$ as a reference structure is intuitively appealing, the SP polarization constants that result have been misinterpreted, introducing a source of error into the predicted values for bound sheet-charge densities (and polarization fields). We also demonstrate that a proper choice of reference structure can eliminate these problems, and we provide revised values that can be directly inserted into current simulation tools.

While our theoretical considerations are general, we choose the nitride semiconductors because they provide a suitable example to illustrate the derivations and because our findings have a significant impact on this materials system of high (and still increasing) technological importance. In Sec. II, we review the underlying theory. In Sec. III, we address the issues resulting from the choice of zincblende as a reference structure, and we propose a solution. Section IV deals with piezoelectric contributions, specifically the issue of proper versus improper constants. In Sec. $\mathrm{V}$, we show that our findings have important 
consequences for nitride device structures and compare with previous implementations and with experiment. Section VI concludes the paper.

\section{CAlCUlating POLARIZATION CONSTANTS IN WURTZITE}

For WZ films grown in the [0001] direction (i.e., the $+c$ direction), the polarization component $P_{3}$ is given by the sum of the SP polarization at the wurtzite material's own lattice parameters, $P_{\mathrm{SP}}$, and the $z$ component of the PZ polarization [1]; for material $m$,

$$
P_{3}^{m}=P_{\mathrm{SP}}^{m}+\left(\epsilon_{1}^{m}+\epsilon_{2}^{m}\right) e_{31}^{m}+\epsilon_{3}^{m} e_{33}^{m},
$$

where (in Voigt notation) $\epsilon_{i}(i=1,2,3)$ is the strain in the $i$ direction and $e_{3 i}$ are the corresponding piezoelectric constants (specifically, the "improper" ones; see Sec. IV). Henceforth, we drop the subscript " 3 " from $P$ for simplicity; all unbolded quantities pertaining to wurtzite are assumed to be in the $c$ direction.

The goal of this work is to derive the appropriate SP and PZ constants that allow Eq. (1) to be used in accurately determining polarization differences at interfaces between different WZ materials.

\section{A. Modern theory of polarization}

Direct calculation of the polarization constants in Eq. (1) by first-principles electronic-structure methods was enabled by the formulation of a rigorous theory of bulk polarization, known as the modern theory of polarization (MTP) $[9,10]$. For a given structure $\lambda$, the MTP allows calculation of the so-called "formal" polarization [9]:

$$
\begin{aligned}
\mathbf{P}_{\mathrm{f}} & =\mathbf{P}_{\mathrm{ion}}+\mathbf{P}_{\mathrm{el}} \\
& =\frac{e}{\Omega} \sum_{s} Z_{s}^{\text {ion }} \mathbf{R}_{s}^{(\lambda)}+\frac{i e f}{8 \pi^{3}} \sum_{j}^{\text {occ }} \int_{\mathrm{BZ}} d \mathbf{k}\left\langle u_{j, \mathbf{k}}^{(\lambda)}\left|\nabla_{\mathbf{k}}\right| u_{j, \mathbf{k}}^{(\lambda)}\right\rangle,
\end{aligned}
$$

where $\Omega$ is the cell volume, $Z_{s}^{\text {ion }}$ is the charge of the ion $s$ and $\mathbf{R}_{\mathrm{s}}^{(\lambda)}$ is its position in the $\lambda$ structure, $f$ is the spin degeneracy of the bands, the sum $j$ runs over occupied bands, and $u_{j, \mathbf{k}}^{(\lambda)}$ are the cell periodic parts of the Bloch wave functions. In Eq. (2), $\mathbf{P}_{\mathrm{el}}$ is the Berry phase taken over the valence-band manifold $[9,10]$. The formal polarization is defined only modulo the "quantum of polarization" $e \mathbf{R} / \Omega$, where $\mathbf{R}$ is any lattice constant and $e$ is the electron charge $[9,10]$.

In the MTP, only differences between formal polarizations of appropriate structures, $\lambda=0$ and $\lambda=1$, are well defined:

$$
\Delta \mathbf{P}=\mathbf{P}_{\mathrm{f}}^{(\lambda=1)}-\mathbf{P}_{\mathrm{f}}^{(\lambda=0)} .
$$

The choice of the "appropriate" structures $\lambda=0$ and $\lambda=1$ rests on one of two possible considerations to ensure that physical conclusions can be drawn from their formal polarization differences. First, if the two structures are connected by an adiabatic, gap-preserving deformation path $[9,10]$, then their difference in polarization $[\Delta \mathbf{P}$ in Eq. (3)] is given by the expression

$$
\Delta \mathbf{P}=\int_{0}^{1} d \lambda \frac{\partial \mathbf{P}}{\partial \lambda},
$$

and it corresponds to the zero-field adiabatic displacement current. This quantity can, in principle, be determined experimentally. An obvious application is the calculation of piezoelectric constants, which involves polarization differences between structures with slightly different lattice constants and/or internal structural parameters.

\section{B. Interface theorem}

The second consideration, as shown by Vanderbilt and King-Smith [11], is that if an insulating interface can be constructed between two structures, the difference in formal polarization gives the bound charge $\sigma_{\mathrm{b}}$ that builds up at the interface as a result of the continuity of the displacement field over an interface with no free charge:

$$
\sigma_{\mathrm{b}}=\left(\mathbf{P}_{\mathrm{f}}^{(\lambda=1)}-\mathbf{P}_{\mathrm{f}}^{(\lambda=0)}\right) \cdot \hat{\mathbf{n}} .
$$

This is often referred to as the "interface theorem." Since there is no adiabatic path necessary between the two structures in this consideration, $\lambda=0$ and $\lambda=1$ can be different polymorphs of the same material (such as WZ and $\mathrm{ZB}$ structures of $\mathrm{GaN}$ ) or different materials altogether (such as GaN and AlN); as long as they form an insulating interface, Eq. (5) will give the bound charge accumulation at the interface.

From the interface theorem [Eq. (5)] and Eq. (1), the bound polarization charge at the interface between different III-nitride materials ( $m$ and $n$ ) is

$$
\begin{aligned}
\sigma_{\mathrm{b}}= & {\left[P_{\mathrm{SP}}^{m}+e_{31}^{m}\left(\epsilon_{1}^{m}+\epsilon_{2}^{m}\right)+e_{33}^{m} \epsilon_{3}^{m}\right] } \\
& -\left[P_{\mathrm{SP}}^{n}+e_{31}^{n}\left(\epsilon_{1}^{n}+\epsilon_{2}^{n}\right)+e_{33}^{n} \epsilon_{3}^{n}\right] .
\end{aligned}
$$

As an example, we take a realistic situation that occurs in heterostructures, assuming that material $n$ is strained coherently to $m\left(\epsilon_{1}^{m}=\epsilon_{2}^{m}=\epsilon_{3}^{m}=0\right)$, i.e., under plane stress $\left(\epsilon_{1}^{n}=\epsilon_{2}^{n}, \epsilon_{3}^{n}=-2 C_{13}^{n} / C_{33}^{n} \epsilon_{1}^{n}\right.$, where $C_{i j}$ are the elastic constants). Therefore, we have

$$
\begin{aligned}
\sigma_{\mathrm{b}} & =\left(P_{\mathrm{SP}}^{m}-P_{\mathrm{SP}}^{n}\right)-2 \epsilon_{1}^{n}\left(e_{31}^{n}-e_{33}^{n} C_{13}^{n} / C_{33}^{n}\right) \\
& =\Delta P_{\mathrm{SP}}^{\mathrm{int}}-2 \epsilon_{1}^{n}\left(e_{31}^{n}-e_{33}^{n} C_{13}^{n} / C_{33}^{n}\right) .
\end{aligned}
$$

Note that $\sigma_{\mathrm{b}}$ is the charge density of electrons at an interface for which material $n$ has been grown on top of material $m$ in the $+c$ direction. 


\section{REFERENCE STRUCTURE FOR SPONTANEOUS POLARIZATION}

\section{A. Effective spontaneous polarization constants}

We first address the difference in spontaneous polarization in Eq. (7), $\Delta P_{\mathrm{SP}}^{\text {int }}$ Strain effects will be taken into account separately in the $\mathrm{PZ}$ part, so that $\Delta P_{\mathrm{SP}}^{\text {int }}$ is simply the difference of formal polarizations of the respective zero-strain structures,

$$
\Delta P_{\mathrm{SP}}^{\mathrm{int}}=\left.P_{\mathrm{f}}^{m}\right|_{\epsilon=0}-\left.P_{\mathrm{f}}^{n}\right|_{\epsilon=0} .
$$

For the purposes of Eq. (1), we would like to define a SP polarization constant that is a property of a single material. Simply taking $P_{\mathrm{f}}^{m}$ of Eq. (8) as $P_{\mathrm{SP}}^{m}$ is problematic since formal polarization is multivalued, being only well defined modulo a quantum of polarization $e \mathbf{R} / \Omega$. Therefore, in every situation in which Eq. (1) is applied to determine $\sigma_{b}$ at an interface, it must be confirmed that formal polarizations of the two materials are taken on the same "branch" of $e \mathbf{R} / \Omega$. A better approach is to take $P_{\mathrm{SP}}^{m}$ in Eq. (1) as a so-called "effective" SP polarization, $\mathbf{P}_{\text {eff }}$, defined by Resta and Vanderbilt [12] to be the $\Delta \mathbf{P}$ in Eq. (3) that results as the system is taken from a high-symmetry "reference" structure $(\lambda=0)$ to the structure of interest $(\lambda=1)$ :

$$
\mathbf{P}_{\text {eff }}=\mathbf{P}_{\mathrm{f}}^{(\lambda=1)}-\mathbf{P}_{\mathrm{f}}^{(\lambda=0)}=\mathbf{P}_{\mathrm{f}}-\mathbf{P}_{\mathrm{f}}^{\text {ref }} .
$$

Using $\mathbf{P}_{\text {eff }}$ to define the SP polarization of the material removes the indeterminacy inherent to the formal polarization.

The reference structure is often chosen to be centrosymmetric, but it is important to recognize that the formal polarization of centrosymmetric crystals is not necessarily zero. This is because, as stated above, $\mathbf{P}_{\mathrm{f}}$ is a multivalued vector field, so it is possible for a nonzero formal polarization to be unchanged (modulo $e \mathbf{R} / \Omega$ ) under the inversion operator. Nevertheless, high symmetry puts restrictions on the possible values of $\mathbf{P}_{f}^{(\lambda=0)}$ [11].

While, in principle, effective polarization constants are still differences in formal polarization between $\lambda=1$ and $\lambda=0$ (reference) structures, in practice they can be used to compare spontaneous polarizations of different materials to obtain $\Delta P_{\mathrm{SP}}$ if such materials share a reference structure with the same formal polarization. Such a comparison then correctly yields the interface charge density according to the interface theorem of Ref. [11]. In such cases, $\Delta P_{\mathrm{SP}}$ is just given by the difference in effective SP polarization of the materials,

$$
\Delta \tilde{P}_{\mathrm{SP}}^{\mathrm{int}}=P_{\mathrm{eff}}^{m}-P_{\mathrm{eff}}^{n} .
$$

In the more general case that the reference formal polarizations do not match, the correct change in SP polarization following from Eqs. (8) and (9) is

$$
\Delta P_{\mathrm{SP}}^{\text {int }}=\Delta \tilde{P}_{\mathrm{SP}}^{\mathrm{int}}+\left(P_{\mathrm{f}}^{m, \text { ref }}-P_{\mathrm{f}}^{n, \text { ref }}\right) .
$$

Therefore, a correction term of the form

$$
\Delta P_{\text {corr }}^{\text {ref }} \equiv P_{\mathrm{f}}^{m, \text { ref }}-P_{\mathrm{f}}^{n, \text { ref }}
$$

has to be added to Eq. (10). Unfortunately, this correction term is not typically implemented in device simulation packages (e.g., Ref. [13]) or used in the interpretation of experimental data (e.g., Ref. [14], which is considered a standard reference in the field).

When the PZ terms are included as well, the total interface charge given by Eq. (7) becomes

$$
\sigma_{\mathrm{b}}=\Delta \tilde{P}_{\mathrm{SP}}^{\mathrm{int}}+\Delta P_{\mathrm{corr}}^{\mathrm{ref}}-2 \epsilon_{1}^{n}\left(e_{31}^{n}-e_{33}^{n} C_{13}^{n} / C_{33}^{n}\right) .
$$

Equation (13) is a central result of the present work.

\section{B. Correction term for the effective spontaneous polarization with the zincblende reference structure}

As mentioned before, previous studies [6-8] have exclusively used $\mathrm{ZB}$ (space group $F \overline{4} 3 m$ ) as a reference structure for calculating the SP polarization of the WZ. This structure is not centrosymmetric, although it has sufficient symmetry to preclude any SP polarization [1]. The fact that an insulating (111) interface can be constructed between the $\mathrm{WZ}$ and $\mathrm{ZB}$ polytypes [8] makes it an appropriate reference structure. In fact, experimental measurements have deduced the relative polarization between the $\mathrm{WZ}$ and $\mathrm{ZB}$ phases of $\mathrm{GaN}$ [5], which were found to be consistent with the theoretical values in Refs. [6-8].

However, there is a subtlety with using $\mathrm{ZB}$ as a reference structure: It has a nonzero formal polarization in the [111] direction, $P_{\mathrm{f}}^{\mathrm{ZB}}$ (modulo $e \mathbf{R} / \Omega$ ). Again, this is consistent with the symmetry considerations because $P_{\mathrm{f}}$ is a multivalued vector quantity and can be nonzero while still remaining unchanged (modulo $e \mathbf{R} / \Omega$ ) under the $F \overline{4} 3 m$ symmetry operations. These symmetry operations dictate the possible values of $P_{\mathrm{f}}^{\mathrm{ZB}}$, and therefore the resulting value depends only on the lattice constant, not on the chemical species of the atoms [11] (see Sec. S1 of Ref. [15] for more details on the formal polarization of ZB). The ZB reference structures for the reported effective SP polarization values for the III-nitrides were those with lattice constants equal to the in-plane lattice constant of the corresponding wurtzite material [6-8] (as confirmed by our calculations), so $P_{\mathrm{f}}^{\mathrm{ZB}}$ will be different for GaN, AlN, and $\mathrm{InN}$, and does not simply constitute a constant shift of $P_{\mathrm{f}}^{\mathrm{WZ}}$ for all the materials. Therefore, for the effective SP polarization constants with the $\mathrm{ZB}$ reference to be implemented in Eq. (7) to determine the polarization difference between different WZ materials, the correction term of Eq. (12) is required, as in Eq. (13).

Consider the example of the interface charge between $\mathrm{InN}$ and $\mathrm{GaN}$. Although, as mentioned above, the formal 
polarization of zincblende does not necessarily vanish, the symmetry of the structure severely limits the possible values. Specifically, there are two possible values of the formal polarization in the [111] direction that are consistent with the symmetry: Either $P_{\mathrm{f}}$ vanishes, or it is equal to $e \sqrt{3} / 2 a_{n}^{2}$ (both modulo $e \sqrt{2} a_{n} / \Omega_{n}$ ), where $a_{n}$ is the WZ in-plane lattice constant of material $n$ and $\Omega_{n}$ is the volume of the ZB primitive cell (see Ref. [11] or Sec. S1 of Ref. [15]). For the III-nitrides, it is the latter, giving a correction term for $\mathrm{GaN} / \mathrm{InN}$ :

$$
\begin{aligned}
\Delta P_{\text {corr }}^{(\mathrm{ZB} \mathrm{ref})} & =P_{\mathrm{f}}^{\mathrm{GaN}, \mathrm{ZB}}-P_{\mathrm{f}}^{\mathrm{InN}, \mathrm{ZB}} \\
& =\frac{e \sqrt{3}}{2}\left(\frac{1}{\left(a_{\mathrm{GaN}}\right)^{2}}-\frac{1}{\left(a_{\mathrm{InN}}\right)^{2}}\right) \\
& =0.28 \mathrm{C} / \mathrm{m}^{2} .
\end{aligned}
$$

When considering the SP polarization differences between WZ nitrides, this represents a significant correction. In fact, as we show in Sec. III C, the correction is an order of magnitude larger than the effective polarizations when they are calculated with the zincblende reference [6,7]. As we see later in Sec. VE, this error is substantially reduced in practice by an approximate error cancellation that occurs in connection with the treatment of the PZ response.

There is nothing intrinsically wrong with the using $\mathrm{ZB}$ as the reference structure for defining WZ effective SP polarization; however, if these values are to be used to obtain polarization differences between different $\mathrm{WZ}$ materials, the $\Delta P_{\text {corr }}^{\text {ref }}$ term [Eq. (12), or Eq. (14) for the example of $\mathrm{GaN} / \mathrm{InN}$ ] must be explicitly included in expressions such as Eq. (11) or Eq. (13). To our knowledge, however, this has not been properly implemented in the numerous previous evaluations of SP polarization for nitride interfaces, and it would require changes in the software for the many simulation tools that include modeling of polarization fields in heterostructures.

\section{C. $P 6_{3} / m m c$ hexagonal layered structure as an alternative reference}

In order to avoid extensive changes in the simulation software, and to enhance physical insight, we advocate another approach, namely, to determine effective SP polarization constants with respect to a reference structure for which the formal polarization is explicitly zero (so that $\left.\Delta P_{\mathrm{SP}}^{\text {int }}=\Delta \tilde{P}_{\mathrm{SP}}^{\mathrm{int}}\right)$. A straightforward choice for this reference structure is the layered hexagonal $(\mathrm{H})$ structure (space group $P 6_{3} / \mathrm{mmc}$ ), as was used for hexagonal $P 6_{3} m c A B C$ materials [16]. This structure is centrosymmetric, and we show below with explicit first-principles calculations that it remains insulating and its formal polarization vanishes. The layered hexagonal structure can be obtained by an adiabatic (gap-preserving) increase of the internal structural $u$ parameter from $u \approx 0.37-0.38$ of the $\mathrm{WZ}$ structure to $u=0.5$. All that is required to avoid correction terms like
Eq. (14) is to replace the effective SP polarization constants currently used in the field (the ones referenced to ZB $[6,7]$ ) with those referenced to the $\mathrm{H}$ reference structure. We have explicitly verified that this leads to expressions that are identical to those that would be obtained for the ZB reference, provided the second term in Eq. (11) or Eq. (13) is included.

The first-principles calculations of $P_{\mathrm{f}}$ for the $\mathrm{H}, \mathrm{WZ}$, and $\mathrm{ZB}$ structures of the III-nitrides were performed using density functional theory with the screened hybrid functional of Heyd, Scuseria, and Ernzerhof (HSE) [17] as implemented in the VASP code [18]. Hartree-Fock mixing parameters of $31 \%$ for $\mathrm{AlN}$ and $\mathrm{GaN}$, and $25 \%$ for $\mathrm{InN}$ were used to correctly describe the band gaps and structural parameters of each material. Conventional functionals based on the local density approximation (LDA) or generalized gradient approximation (GGA) predict $\mathrm{InN}$ to be a metal, precluding the calculation of the polarization constants if the $\Gamma$ point is included in the $k$-point mesh (which is required in Vasp). Projector augmented wave potentials (PAW) [19], with the In and Ga $d$ electrons frozen in the core, were used. All calculations were performed on bulk primitive cells, with a $6 \times 6 \times 8$ Monkhorst-Pack [20] $k$-point mesh to sample the Brillouin zone, and a large energy cutoff of $600 \mathrm{eV}$ for the plane-wave basis set, chosen to ensure convergence of the internal structural parameter $u$. The calculated lattice parameters and band gaps, listed in Sec. S2 of Ref. [15], show good agreement with experimental data.

We have calculated the electronic structure for structures with increasing $u$, ranging from $u \approx 0.37$ to $u=0.5$ (Fig. 1), and confirmed that this path between $\mathrm{WZ}$ and

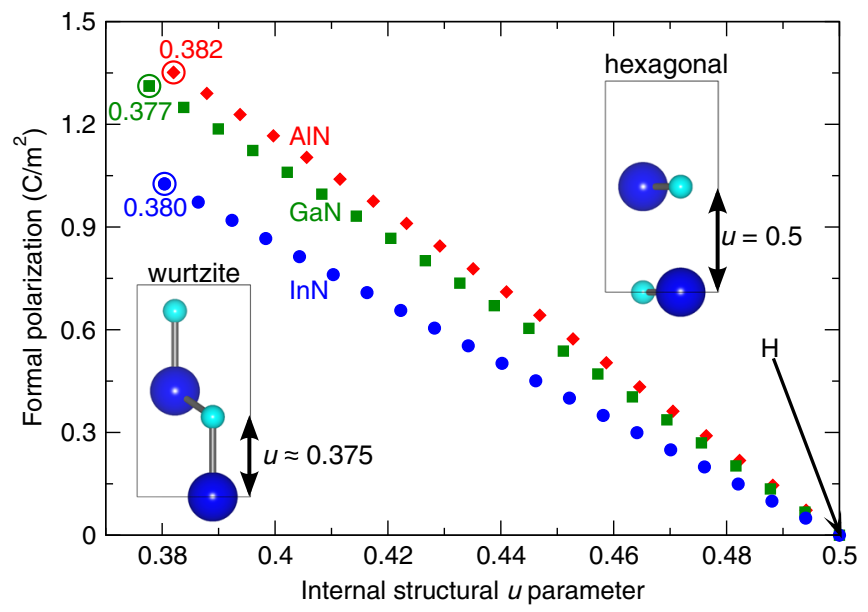

FIG. 1. Formal polarization of InN, GaN, and AlN for structures as a function of the internal structural parameter $u$, varying between fully relaxed WZ (circled symbols, labeled with the relaxed $u$ value) and $\mathrm{H}(u=0.5)$, as shown schematically by ball and stick models, where smaller balls represent $\mathrm{N}$ atoms and larger ones represent the cations. All other lattice parameters were fixed at their relaxed $\mathrm{WZ}$ values. 
$\mathrm{H}$ is gap preserving. These calculations also show that the formal polarization of the $\mathrm{H}$ structure is zero (modulo $e \mathbf{R} / \Omega$ ) for the III-nitrides (Fig. 1). We remind the reader that this was not guaranteed since $\mathbf{P}_{\mathrm{f}}$ can be nonzero and still consistent with inversion symmetry, if the inversion operator changes $\mathbf{P}_{\mathrm{f}}$ by a multiple of $e \mathbf{R} / \Omega$. We have therefore verified that the hexagonal phase is a reference structure for which there is no spurious term in Eq. (11).

In addition, by correcting for any discontinuities (in the amount of a multiple of $e \mathbf{R} / \Omega$ ) that may occur in the calculations of formal polarizations along the path between $\mathrm{WZ}$ and $\mathrm{H}$, we have ensured that we are comparing formal polarizations of $\mathrm{WZ} \mathrm{GaN}, \mathrm{AlN}$, and $\mathrm{InN}$ on the same branch of $e \mathbf{R} / \Omega$ [12].

The calculated spontaneous polarization coefficients for the WZ structure using either $\mathrm{H}$ or $\mathrm{ZB}$ as a reference are given in Table I. The results obtained by Bernardini et al. $[6,7]$ are listed for comparison. The GGA functional used in that work provides results that are very close to those we obtained with HSE; the discrepancy is the largest for InN, which is probably related to the fact, mentioned above, that GGA predicts InN to be a metal. Table I also shows, however, that the choice of reference structure makes a significant difference. The magnitudes of the coefficients are much larger, and their signs are different when $\mathrm{H}$ is used as the reference. We observe that it is not just the absolute values but also the relative differences between the calculated polarization constants of the three materials that differ from the previously reported values [6,7].

The difference in sign of $P_{\mathrm{eff}}^{(\mathrm{H} \text { ref })}$ compared to $P_{\mathrm{eff}}^{(\mathrm{ZB} \text { ref })}$ demonstrates that the conventional wisdom that the SP polarization in $\mathrm{WZ}$ points in the $-c$ direction is misleading. The formal polarization of WZ has no definite sign as this would depend on the chosen branch. The effective SP depends on the polarization difference, and therefore, the sign will depend on the sign and magnitude of the formal polarization of the reference structure.

Even though the values reported in Table I for $P_{\text {eff }}^{(\mathrm{Href})}$ and $P_{\text {eff }}^{\text {(ZB ref) }}$ clearly differ in sign, absolute magnitude, and

TABLE I. Effective spontaneous polarization constants in units of $\mathrm{C} / \mathrm{m}^{2}$ of $\mathrm{WZ} \mathrm{GaN}, \mathrm{AlN}$, and $\mathrm{InN}$ calculated using either the hexagonal $\left(\mathrm{H}\right.$, space group $\left.P 6_{3} / m m c\right)$ or $\mathrm{ZB}$ (space group $F \overline{4} 3 m$ ) reference structures. The lattice constant of the $\mathrm{ZB}$ structure is chosen to match the in-plane lattice constant of the WZ structure for the same material. Results from previous calculations [7] that used the $\mathrm{ZB}$ reference are listed for comparison.

\begin{tabular}{lccc}
\hline \hline & $P_{\text {eff }}^{(\mathrm{H} \text { ref })}$ & $P_{\text {eff }}^{\text {(ZB ref })}$ & $P_{\text {eff }}^{(\text {ZB ref })}$, previously reported \\
\hline GaN & 1.312 & -0.035 & -0.034 \\
AlN & 1.351 & -0.090 & -0.090 \\
InN & 1.026 & -0.053 & -0.042 \\
\hline \hline
\end{tabular}

${ }^{\mathrm{a}}$ From Ref. [7]. relative differences between materials, we show in Sec. V that the final predictions based on both formulations are actually rather similar because of the way the PZ contributions have been included in the previous work (cf. Sec. IV).

\section{IMPROPER VERSUS PROPER PIEZOELECTRIC CONSTANTS}

We now address the specifics of the PZ terms in Eqs. (7) and (13). A complication that must be addressed is the choice between improper and proper $e_{31}\left(e_{33}\right.$ has no such complication) $[2,4]$.

As we have done above, we consider a thin layer of a WZ material grown in the $c$ direction. If the layer is strained perpendicular to the $c$ direction, the total bound charge on the $+c$ and $-c$ surfaces will change as a result of the polarization current, or redistribution of charge, in the layer. If metallic contacts on the $+c$ and $-c$ surfaces are shortcircuited when the strain occurs, the current flow can be measured directly and will give the proper PZ constant, denoted $e_{31}^{\text {prop }}[2,4]$.

If the $+c$ and $-c$ faces are in open-circuit boundary conditions, the layer will have a field across it due to the SP polarization, which will be modified by the strain via two mechanisms. The first is the same as in the proper case, as the strain will cause a flow of polarization current. But in addition, since the field depends on the charge density, the change in the area of the $c$ plane as a result of $\epsilon_{1}$ will dilute or concentrate the "pre-strain" bound charge. For small strains, the latter is given by the zero-strain formal polarization [2,4]. Taking both of these mechanisms into account gives the improper PZ constant, $e_{31}^{\mathrm{imp}}$.

In the case of, e.g., Eq. (13), the PZ constants correspond to the improper case since their role in the equation is to take into account the change in formal polarization of material $n$ with strain, so that $\sigma_{b}$ corresponds to the bound charge at the coherent interface with the in-plane lattice constant of material $m$. The change in formal polarization with strain is an alternative definition of the improper PZ constants [4].

From Refs. [2] and [4], the improper PZ constant $e_{31}^{n \text {,imp }}$ is related to the proper constant by

$$
e_{31}^{n, \text { imp }}=e_{31}^{n, \text { prop }}-\left.P_{\mathrm{f}}^{n}\right|_{\epsilon=0},
$$

where $\left.P_{\mathrm{f}}^{n}\right|_{\epsilon=0}$ is the zero-strain formal polarization of material $n$. There is no change to the $e_{33} \mathrm{PZ}$ constant. The proper PZ constant is a well-defined bulk quantity, as it is related to the polarization current; however, the improper PZ constant is branch dependent [4]. Here, also, defining polarization with respect to the $\mathrm{H}$ reference proves useful. Since the formal polarization of the $\mathrm{H}$ structure vanishes (Fig. 1), $P_{\text {eff }}^{n,(\mathrm{H} r e f)}=\left.P_{\mathrm{f}}^{n, \mathrm{WZ}}\right|_{\epsilon=0}$; this also ensures that improper PZ constants for the different materials are 
taken on the same branch, in the same way as this is confirmed for the SP polarization constants. Therefore, consistent use of the $\mathrm{H}$ reference structure allows us to write Eq. (7) as

$$
\begin{aligned}
\sigma_{\mathrm{b}}= & \Delta \tilde{P}_{\mathrm{SP}}^{\text {int,(H ref })} \\
& -2 \epsilon_{1}^{n}\left(e_{31}^{n, \text { prop }}-P_{\mathrm{eff}}^{n,(\mathrm{H} \mathrm{ref})}-e_{33}^{n, \text { prop }} C_{13}^{n} / C_{33}^{n}\right),
\end{aligned}
$$

where $P_{\text {eff }}^{n,(\mathrm{H} \text { ref })}$ can be taken from Table I.

Calculated proper PZ constants are given in the "proper" column of Table II. Since the HSE hybrid functional was used (and therefore density functional perturbation theory was not implemented), finite differences were used to calculate the derivatives with strain, following the procedure outlined in Eqs. (4)-(6) in Ref. [6]. Specifically, improper PZ constants were calculated and converted to proper constants by adding $\left.P_{\mathrm{f}}^{n}\right|_{\epsilon=0}$ [see Eq. (15)] as determined in the calculation. This removes any dependence on the branch choice used in finite-difference calculations [4]. We then convert back to improper constants using $P_{\text {eff }}^{n,(\text { H ref })}$ as discussed above, in order to ensure that the constants are reported for the same branch for each material ("improper" column in Table II).

The WZ structure does have another nonzero piezoelectric constant $e_{15}$, which couples a shear deformation in a plane perpendicular to the $c$ plane $\left(\epsilon_{13}\right.$ or $\left.\epsilon_{31}\right)$ to the polarization in the $c$ plane. In this case, there are two improper PZ constants since a correction must be included in the case of $\epsilon_{13}$ but not for $\epsilon_{31}$ [4]. These elements do not enter in the situation we consider in this work (plane stress conditions with the $c$ plane as the growth plane) but may be important for growth on nonpolar or semipolar planes [21].

It is important to comment on the PZ constants reported in the literature [3]. When PZ polarization constants have been implemented in simulations (e.g., Refs. $[3,13,14])$, it has never been specified which PZ constants are used for WZ III-nitrides. However, by comparing our calculations of proper and improper PZ constants ("Proper" and "Improper" columns of Table II) with the reported PZ constants in the literature ("Previously reported" of Table II), we have found that the reported constants are more likely to be the proper PZ constants.

TABLE II. Calculated piezoelectric polarization constants in units of $\mathrm{C} / \mathrm{m}^{2}$ compared with reported values from the literature.

\begin{tabular}{llrcc}
\hline \hline & & Proper & Improper & Previously reported $^{\text {a }}$ \\
\hline $\mathrm{GaN}$ & $e_{31}$ & -0.551 & -1.863 & -0.22 to -0.55 \\
& $e_{33}$ & 1.020 & 1.020 & 0.43 to 1.12 \\
$\mathrm{AlN}$ & $e_{31}$ & -0.676 & -2.027 & -0.38 to -0.81 \\
& $e_{33}$ & 1.569 & 1.569 & 1.29 to 1.94 \\
$\mathrm{InN}$ & $e_{31}$ & -0.604 & -1.63 & -0.23 to -0.59 \\
& $e_{33}$ & 1.238 & 1.238 & 0.39 to 1.09 \\
\hline \hline
\end{tabular}

${ }^{\mathrm{a}}$ From Ref. [3] and references therein.
From an experimental perspective, most of the experimental techniques have measured total polarization and then deduced the PZ constants in the nitrides using the SP constants from, e.g., Ref. [6] using Eq. (1). As we show in Sec. V, observation of the effects of total polarization can be misleading with regards to the differentiation between proper and improper PZ constants because of the error cancellation from the use of the $\mathrm{ZB}$ reference in defining the SP polarization (discussed in Sec. III B).

There have been direct measurements of the PZ constants, either by probing the electromechanical coupling constants via surface acoustic waves [22,23] or by using interferometry to determine the strain caused by the application of a voltage [24-27]. Both of these techniques measure the proper constants since neither is sensitive to the change in surface charge density resulting from the deformation. These reported values indeed agree well with our calculated values for the proper PZ constants, both in sign and in magnitude.

In previous work [7], $P_{\text {eff }}^{n, \text { (ZB ref) }}$ was used instead of $\left.P_{\mathrm{f}}^{n}\right|_{\epsilon=0}$ in Eq. (15) to convert improper to proper $e_{31} \mathrm{PZ}$ constants (cf. Tables VI and V of Ref. [7]). Because of the nonvanishing formal polarization of the $\mathrm{ZB}$ reference structure, $P_{\text {eff }}^{n, \text { (ZB ref) }} \neq\left. P_{\mathrm{f}}^{n, \mathrm{WZ}}\right|_{\epsilon=0}$; instead, we see from the discussion resulting in Eq. (14) that Eq. (15) can be expressed as

$$
e_{31}^{n, \text { imp }}=e_{31}^{n, \text { prop }}-\left(P_{\mathrm{eff}}^{n, \text { (ZB ref })}+\frac{e \sqrt{3}}{2 a_{n}^{2}}\right)
$$

where $a_{n}$ is the equilibrium, in-plane lattice constant of the $\mathrm{WZ}$ material $n$. To our knowledge, the inclusion of the last term in Eq. (17) has not been discussed in the literature. Because of the small magnitude of $P_{\mathrm{eff}}^{n, \text { (ZB ref) }}$, neglecting the last term in Eq. (17) led to the conclusion in Ref. [7] that the difference between the proper and improper PZ constants is small, seemingly rendering the distinction of no consequence. Instead, because of the large magnitude of $P_{\mathrm{eff}}^{n,(\mathrm{H} \text { ref })}$ [and $e \sqrt{3} / 2 a_{n}^{2}$ in Eq. (17)], the distinction between proper and improper PZ constants is actually very significant.

\section{COMPARISON WITH REPORTED EXPERIMENTAL RESULTS}

\section{A. Correct expressions for total polarization for wurtzite materials}

Before discussing specific quantitative results for nitride semiconductors, we briefly summarize the main points of the previous sections and rigorously express the polarization of a given WZ material [Eq. (1)]. Spontaneous polarization constants must be defined with respect to a reference structure, and this choice of reference structure must be taken into account when evaluating polarization discontinuities at interfaces. We determined a correction 
term [Eq. (12)] that is necessary when effective SP polarization constants are used to determine the SP polarization difference between materials at an interface. This correction term is significant when the $\mathrm{ZB}$ reference structure is used [e.g., Eq. (14)] but is zero for the $\mathrm{H}$ reference. Using $\mathrm{H}$ as a reference is therefore more straightforward and is the approach we advocate, with the SP constants $P_{\text {eff }}^{(\mathrm{H} \text { ref })}$ listed in Table II.

In addition, the improper PZ constants should be used to determine interface bound charge and fields in heterostructure layers. These can be obtained from the proper constant $e_{31}^{\text {prop }}$ by subtracting $P_{\text {eff }}^{(\mathrm{H} \text { ref })}$ (Table II). Therefore, in the notation of this paper, Eq. (1) is written rigorously as

$P=P_{\text {eff }}^{(\text {H ref })}+\left(\epsilon_{1}+\epsilon_{2}\right)\left(e_{31}^{\text {prop }}-P_{\text {eff }}^{(\mathrm{H} \mathrm{ref})}\right)+\epsilon_{3} e_{33}^{\text {prop }}$.

\section{B. Calculation of sheet charges for III-nitrides}

Because of the important impact of polarization on device performance and design, a plethora of experimental studies have been aimed at determining the effects of polarization at $\mathrm{GaN} / \mathrm{InGaN}$ and GaN/AlGaN heterostructures. We have plotted these reported results in Fig. 2, expressed as the magnitude of polarization bound charge at the interface, as a function of alloy content (a full list of references is provided in Sec. S3 of Ref. [15]).

For $\mathrm{GaN}$ grown in the $+c$ direction with the $\mathrm{InGaN}$ $(\mathrm{AlGaN})$ grown on top, the sign of the bound charge at the interface will be negative (positive) [28].

In Fig. 2, the black dashed curves correspond to the current practice in the field: Sheet charges are predicted based on (i) SP constants referenced to the ZB structure $\left(P_{\text {eff }}^{\text {(ZB ref) }}\right.$ in Table I) and Eq. (13) without the correction term $\Delta P_{\text {corr }}^{\text {(ZB) }}$, and (ii) proper PZ constants ("Proper" column in Table II). Quantities for alloys were obtained using linear interpolation. For an explicit expression in terms of alloy content, see Eq. (3) in Sec. S4 of Ref. [15]. Elastic constants were taken from Ref. [29].

The red solid line in Fig. 2 corresponds to the implementation recommended in this work, i.e., using the $\mathrm{H}$ reference structure and the improper PZ constants, as in Eqs. (16) and (18) [and Eq. (5) in Sec. S4 of Ref. [15]].

In view of the arguments given above, it may seem surprising that the dashed black and solid red curves agree as well as they do; we return to this point in Sec. V E.

\section{InGaN/GaN interfaces}

For the InGaN/GaN system, most experimental studies have applied optical techniques to determine the polarization fields in $\mathrm{GaN} / \mathrm{InGaN} / \mathrm{GaN}$ quantum wells (QWs). This field can be probed by varying the QW width [30] or external bias [31] and measuring the change in the optical properties of the QW (labeled "optical" in Fig. 2).
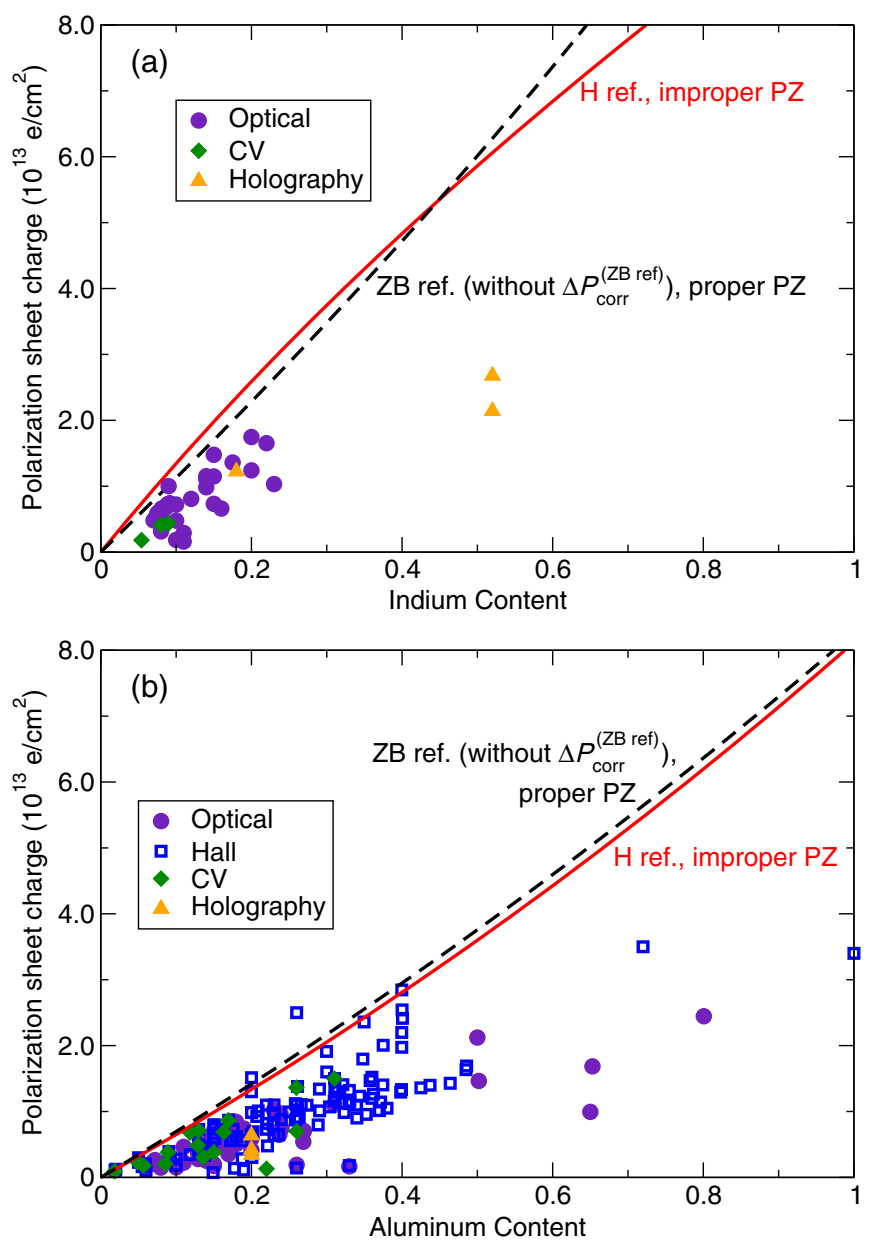

FIG. 2. Absolute values for polarization sheet charges at the (a) $\mathrm{InGaN} / \mathrm{GaN}$ and (b) $\mathrm{AlGaN} / \mathrm{GaN}$ interfaces as a function of alloy content predicted from the spontaneous polarization constants calculated using either the ZB reference structure [without a correction term, Eq. (14)] and the proper piezoelectric constants (black dashed curve), or the $\mathrm{H}$ reference structure and improper piezoelectric constants (red solid curve). Points are experimental values from the literature (see Sec. S3 of Ref. [15] for references and values).

In addition, there have also been studies using timeresolved PL to measure shifts due to screening of the polarization field by photoexcited or electrically injected carriers [32]. Other studies have been based on capacitance-voltage (CV) profiling of the fields [33], and on electron holography [34], where cross-sectional transmission electron microscopy is conducted on InGaN/GaN heterostructures to determine the depthresolved electrostatic potential in the growth direction. When fields are reported, we convert to the bound charge for the purposes of Fig. 2(a), assuming GaN/InGaN/GaN quantum wells (with thick barriers such that the electric field in the barriers is presumed to be zero) using a simple parallel-plate capacitor model $\left(E=\sigma / \varepsilon_{0} \varepsilon_{r}\right.$, using a relative dielectric constant for $\mathrm{GaN}$ of 10 [35] and for $\mathrm{InN}$ of 15 [36] and a linear interpolation for the dielectric 
constant of InGaN). For specific values of the points in Fig. 2, see Sec. S3 of Ref. [15].

The red curve in Fig. 2(a) is indeed in reasonable agreement with the experimental observations, appearing to be an upper bound of the data. The optical experiments usually rely on Schrödinger-Poisson simulations to determine the field magnitude from the measured optical properties. Uncertainties in input parameters to these models such as well widths, compositions, and composition profiles can result in quantitative differences. It has been shown recently that taking into account the deviations from an ideal QW structure when interpreting experimental observations can account for the apparent discrepancy between the measurements and theoretical prediction of polarization fields [37,38]. Such deviations are expected to be significant for InGaN/GaN because of the large lattice mismatch and the large difference in optimal growth temperatures for $\mathrm{GaN}$ and $\mathrm{InGaN}$.

\section{AlGaN/GaN interfaces}

For the $\mathrm{AlGaN} / \mathrm{GaN}$ system, there are two basic strategies for experimentally determining polarization effects. The first is to directly measure the polarization field in an AlGaN/GaN/AlGaN (QW) structure with the same methods as used in the InGaN/GaN case [39,40]. For the purposes of Fig. 2(b), we have converted these fields into bound sheet-charge densities in an AlGaN/GaN/AlGaN quantum well (using a relative dielectric constant for $\mathrm{GaN}$ of 10 [35]).

The other strategy is to measure the density of the 2DEG at the $\mathrm{AlGaN} / \mathrm{GaN}$ interface in a HEMT structure ( $\mathrm{GaN}$ channel, $\mathrm{AlGaN}$ barrier); from this, the bound interface charge $\sigma_{\mathrm{b}}$ can be derived [41]. The 2DEG density can be determined either by Hall effect [14] or CV [41] measurements.

The significant scatter in the experimental data in Fig. 2(b) may have several origins. There are experimental uncertainties that can influence fields, such as incomplete strain relaxation in buffer layers [42] and differences in background doping [43-45].

As in the case of InGaN/GaN, the predicted sheet charges appear to be an overestimation compared to the experimental observations. In the case of optical measurements, Schrödinger-Poisson modeling is again typically used to interpret the measured properties, and the same uncertainties and systematic errors may arise as discussed in the case of InGaN/GaN [46,47]. For the cases where the compensating 2DEG density is measured, the thickness of the AlGaN layer and Schottky barrier height at the AlGaN surface will determine whether the entire bound charge is compensated, which could be a reason that the observations are slightly lower than predicted theoretically [48]. Also, interface roughness and electron traps due to dislocations and/or surface states have been proposed to explain the reduced 2DEG density [14].

\section{E. Comparison of theoretical implementations}

The degree of agreement between results obtained based on the current practice in the field (ZB reference, no correction term, proper PZ) and our revised implementation (H reference, improper PZ constants) merits some discussion. It is clear from Table I that the SP polarization values determined with the $\mathrm{H}$ reference structure are very different from those determined with the ZB reference; also, from Table II, the improper and proper $e_{31}$ coefficients are very different. However, the similarity between the red solid and black dashed curves in Fig. 2 demonstrates that these two large corrections cancel when we evaluate Eq. (18). In other words, the correct implementation (H reference, improper PZ constants) results in values that are only slightly different from the current (incorrect) practice in the field (ZB reference, no correction term, proper PZ). We now demonstrate analytically how this accidental agreement comes about.

If we take the difference between our revised implementation (solid red curve in Fig. 2) and the current practice in the field (dashed black curve in Fig. 2) for a given $x$, we obtain the total error in using the current practice in the field:

$$
\Delta P_{\text {error }}=x \Delta P_{\text {corr }}^{\text {(ZB ref })}+2 \epsilon_{1}(x) P_{\text {eff }}^{n,(\mathrm{H} \mathrm{ref})}(x) .
$$

A derivation of this expression is given in Sec. S5 of Ref. [15]. For both $\mathrm{AlGaN} / \mathrm{GaN}$ and $\mathrm{InGaN} / \mathrm{GaN}$, the two terms on the right-hand side of Eq. (19) have opposite signs and a tendency to cancel. To understand why, first note that $\epsilon_{1}(x)$ is approximately linear in $x$, so that both terms can be regarded as being roughly proportional to $\epsilon_{1}(x)$. In particular, linearizing Eq. (14) in $\epsilon_{1}$, we find $x \Delta P_{\text {corr }}^{\text {(ZB ref })} \simeq$ $-2 \epsilon_{1}(x) P_{\mathrm{f}}^{m, \mathrm{ZB}}$ [see also Eq. (8) of Ref. [15]]. Thus,

$$
\begin{aligned}
\Delta P_{\mathrm{error}} & \simeq 2 \epsilon_{1}(x)\left(P_{\mathrm{eff}}^{n,(\mathrm{H} \text { ref })}-P_{\mathrm{f}}^{m, \mathrm{ZB}}\right) \\
& =2 \epsilon_{1}(x)\left(P_{\mathrm{f}}^{n, \mathrm{WZ}}-P_{\mathrm{f}}^{m, \mathrm{ZB}}\right) \\
& =2 \epsilon_{1}(x)\left[P_{\mathrm{eff}}^{n, \mathrm{ZB} \text { ref })}+\left(P_{\mathrm{f}}^{n, \mathrm{ZB}}-P_{\mathrm{f}}^{m, \mathrm{ZB}}\right)\right],
\end{aligned}
$$

where in the second step we used the fact that the formal polarization of the $\mathrm{H}$ structure vanishes. For the III-nitrides, $\left|\epsilon_{1}\right|<0.1$, and we see from Table I that $\left|P_{\text {eff }}^{n, \mathrm{ZB}}\right|<0.1 \mathrm{C} / \mathrm{m}^{2}$. The second term in the last line of Eq. (20), $P_{\mathrm{f}}^{n, \mathrm{ZB}}-P_{\mathrm{f}}^{m, \mathrm{ZB}}$, is related to the difference in inplane lattice constants between material $n$ and material $m$ (Sec. III B). The largest value it will take for the materials considered in this study is $0.28 \mathrm{C} / \mathrm{m}^{2}$ for $\mathrm{InN}$ on $\mathrm{GaN}$, as calculated in Eq. (14), and it will be significantly smaller for lower alloy content and for the case of $\mathrm{AlGaN}$ on $\mathrm{GaN}$. The error is therefore the product of small factors, and thus small in practice, significantly smaller than the errors in the SP and PZ parts individually. 


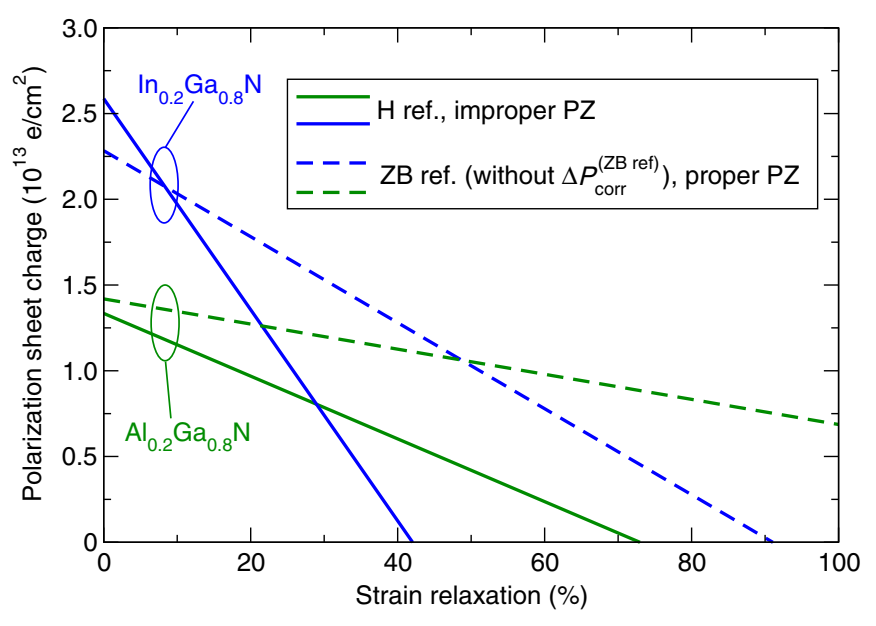

FIG. 3. Absolute values for polarization sheet charges at the $\mathrm{In}_{0.2} \mathrm{Ga}_{0.8} \mathrm{~N} / \mathrm{GaN}$ (blue) and $\mathrm{Al}_{0.2} \mathrm{Ga}_{0.8} \mathrm{~N} / \mathrm{GaN}$ (green) interface as a function of percent strain relaxation. Here, $0 \%$ relaxation corresponds to perfectly strained layers, and $100 \%$ relaxation to an unstrained overlayer at its bulk lattice constant. Solid curves are the correct implementation described in this work (H reference for SP and improper PZ constants); dashed curves are the current practice in the field (ZB reference for SP, without the correction term, and proper PZ constants).

The small magnitude of $P_{\text {eff }}^{n, \mathrm{ZB}}$ (Table I) demonstrates that $P_{\mathrm{f}}^{\mathrm{WZ}} \sim P_{\mathrm{f}}^{\mathrm{ZB}}$ in these materials (see Sec. S1 of Ref. [15]). The similarity between $P_{\mathrm{f}}^{\mathrm{ZB}}$ and $P_{\mathrm{f}}^{\mathrm{WZ}}$ is not unexpected. Although $\mathrm{ZB}$ has sufficient symmetry to preclude SP polarization, in the [111] direction the structure only differs from the WZ $c$ direction by the stacking of the cation/anion planes and a small deviation from the ideal WZ $u$ parameter and $c / a$ lattice constants. In WZ materials other than the III-nitrides, such deviations could, in principle, be larger, and in that case, $P_{\text {eff }}^{\mathrm{ZB} \text { ref }}$ will be larger, resulting in $\Delta P_{\text {error }}$ being more significant.

For $\mathrm{AlGaN} / \mathrm{GaN}$, the relatively modest difference in lattice constants between $\mathrm{AlN}$ and $\mathrm{GaN}$ (and therefore modest $\epsilon_{1}$ values for coherently strained alloy layers), and an almost exact cancellation between $P_{\mathrm{f}}^{\mathrm{GaN}, \mathrm{ZB}}$ and $P_{\text {eff }}^{\mathrm{AlGaN},(\mathrm{H} \text { ref) }}$ means that the difference between implementations is small over the whole composition range (cf. Sec. S5 of Ref. [15]). For InGaN/GaN, the large lattice mismatch of InN and $\mathrm{GaN}$ and a less complete cancellation of $P_{\mathrm{f}}^{\mathrm{GaN}, \mathrm{ZB}}$ and $P_{\mathrm{eff}}^{\mathrm{InGaN},(\mathrm{H} \mathrm{ref})}$ results in a significant deviation at higher In content; this will be important for the prediction of polarization fields in applications such as tunnel field-effect transistors based on thin, high In-content interlayers [49].

The two implementations differ significantly in the relative contributions of $\mathrm{SP}$ and $\mathrm{PZ}$ polarization. An effect of this is illustrated by the case where there is strain relaxation in the alloy layer. In Fig. 3, the predicted polarization bound charges for $\operatorname{In}_{0.2} \mathrm{Ga}_{0.8} \mathrm{~N} / \mathrm{GaN}$ (blue curves) and $\mathrm{Al}_{0.2} \mathrm{Ga}_{0.8} \mathrm{~N} / \mathrm{GaN}$ (green curves) are shown as a function of strain relaxation of the layer, modeled by simply scaling $\epsilon_{1}$. For both InGaN/GaN and $\mathrm{AlGaN} / \mathrm{GaN}$, the revised implementation of this work predicts a much faster decrease in bound charge at the interface than the current practice in the field. Of course, strain relaxation is associated with the presence of edge dislocations at the interface, which may themselves influence the interface bound charge; this effect has not been taken into account in either version of the implementation.

\section{CONCLUSIONS}

We have derived a rigorously correct implementation of polarization constants in wurtzite materials, focusing on the example of the III-nitrides. Our derivation has demonstrated the impact of the choice of reference structure when calculating spontaneous polarization constants using the modern theory of polarization. Insufficient care in using the values can result in spurious contributions to the polarization discontinuities at heterostructure interfaces. We have provided new values calculated with a consistent hexagonal (rather than zincblende) reference structure. In addition, we have demonstrated the importance of choosing the correct piezoelectric constants (improper) and provided values for these improper constants. These revised values of the spontaneous and piezoelectric constants can be directly used in simulations and to interpret experimental observations. The revised implementation predicts a more rapid decrease of polarization charge with strain relaxation for an alloy layer on $\mathrm{GaN}$.

\section{ACKNOWLEDGMENTS}

Work by C. E. D. was supported by the U.S. Department of Energy (DOE), Office of Science, Basic Energy Sciences (BES) under Award No. DE-SC0010689. Work by A. J. was supported by the Center for Low Energy Systems Technology (LEAST), one of the six SRC STARnet Centers, sponsored by MARCO and DARPA. Work by D. V. was supported by ONR Grant No. N00014-12-11035. Computational resources were provided by the CSC at the CNSI and MRL (an NSF MRSEC, DMR-1121053) (NSF CNS-0960316) and by the National Energy Research Scientific Computing Center, a DOE Office of Science User Facility supported by the Office of Science of the U.S. Department of Energy under Contract No. DE-AC02$05 \mathrm{CH} 11231$.

[1] J. F. Nye, Physical Properties of Crystals (Oxford University Press, London, 1957), p. 300.

[2] R. M. Martin, Piezoelectricity, Phys. Rev. B 5, 1607 (1972).

[3] M. Feneberg and K. Thonke, Polarization Fields of III-Nitrides Grown in Different Crystal Orientations, J. Phys. Condens. Matter 19, 403201 (2007). 
[4] D. Vanderbilt, Berry-Phase Theory of Proper Piezoelectric Response, J. Phys. Chem. Solids 61, 147 (2000).

[5] J. Lähnemann, O. Brandt, U. Jahn, C. Pfüller, C. Roder, P. Dogan, F. Grosse, A. Belabbes, F. Bechstedt, A. Trampert, and L. Geelhaar, Direct Experimental Determination of the Spontaneous Polarization of GaN, Phys. Rev. B 86, 081302 (2012).

[6] F. Bernardini, V. Fiorentini, and D. Vanderbilt, Spontaneous Polarization and Piezoelectric Constants of III-V Nitrides, Phys. Rev. B 56, R10024 (1997).

[7] F. Bernardini, V. Fiorentini, and D. Vanderbilt, Accurate Calculation of Polarization-Related Quantities in Semiconductors, Phys. Rev. B 63, 193201 (2001).

[8] F. Bechstedt, U. Grossner, and J. Furthmüller, Dynamics and Polarization of Group-III Nitride Lattices: A FirstPrinciples Study, Phys. Rev. B 62, 8003 (2000).

[9] R. D. King-Smith and D. Vanderbilt, Theory of Polarization of Crystalline Solids, Phys. Rev. B 47, 1651 (1993).

[10] R. Resta, Macroscopic Polarization in Crystalline Dielectrics: The Geometric Phase Approach, Rev. Mod. Phys. 66, 899 (1994).

[11] D. Vanderbilt and R. D. King-Smith, Electric Polarization as a Bulk Quantity and Its Relation to Surface Charge, Phys. Rev. B 48, 4442 (1993).

[12] R. Resta and D. Vanderbilt, in Physics of Ferroelectrics: A Modern Perspective, edited by K. Rabe, Ch. H. Ahn, and J.-M. Triscone (Springer-Verlag, Berlin, 2007).

[13] SiLENSe Physics Summary v5.2 (2011).

[14] O. Ambacher, B. Foutz, J. Smart, J. R. Shealy, N. G. Weimann, K. Chu, M. Murphy, R. Dimitrov, A. Mitchell, and M. Stutzmann, Two Dimensional Electron Gases Induced by Spontaneous and Piezoelectric Polarization in Undoped and Doped AlGaN/GaN Heterostructures, J. Appl. Phys. 87, 334 (2000).

[15] See Supplemental Material at http://link.aps.org/ supplemental/10.1103/PhysRevX.6.021038 for a discussion of formal polarization in $\mathrm{ZB}$, a table of structural properties and band gaps, a list of experimental references, equations used to generate curves in Fig. 2, and equations demonstrating the difference between implementations.

[16] J. W. Bennett, K. F. Garrity, K. M. Rabe, and D. Vanderbilt, Hexagonal ABC Semiconductors as Ferroelectrics, Phys. Rev. Lett. 109, 167602 (2012).

[17] J. Heyd, G. E. Scuseria, and M. Ernzerhof, Hybrid Functionals Based on a Screened Coulomb Potential, J. Chem. Phys. 118, 8207 (2003); 124, 219906 (2006).

[18] G. Kresse and J. Furthmüller, Efficient Iterative Schemes for Ab Initio Total-Energy Calculations Using a Plane-Wave Basis Set, Phys. Rev. B 54, 11169 (1996).

[19] P. E. Blöchl, Projector Augmented-Wave Method, Phys. Rev. B 50, 17953 (1994).

[20] H. J. Monkhorst and J. D. Pack, Special Points for BrillouinZone Integrations, Phys. Rev. B 13, 5188 (1976).

[21] A. E. Romanov, T. J. Baker, S. Nakamura, and J. S. Speck, Strain-Induced Polarization in Wurtzite III-Nitride Semipolar Layers, J. Appl. Phys. 100, 023522 (2006).

[22] G. D. O'Clock and M. T. Duffy, Acoustic Surface Wave Properties of Epitaxially Grown Aluminum Nitride and Gallium Nitride on Sapphire, Appl. Phys. Lett. 23, 55 (1973).
[23] M.-A. Dubois and P. Muralt, Properties of Aluminum Nitride Thin Films for Piezoelectric Transducers and Microwave Filter Applications, Appl. Phys. Lett. 74, 3032 (1999).

[24] S. Muensit and I. L. Guy, The Piezoelectric Coefficient of Gallium Nitride Thin Films, Appl. Phys. Lett. 72, 1896 (1998).

[25] S. Muensit, E. M. Goldys, and I. L. Guy, Shear Piezoelectric Coefficients of Gallium Nitride and Aluminum Nitride, Appl. Phys. Lett. 75, 3965 (1999).

[26] I. L. Guy, S. Muensit, and E. M. Goldys, Extensional Piezoelectric Coefficients of Gallium Nitride and Aluminum Nitride, Appl. Phys. Lett. 75, 4133 (1999).

[27] C. M. Lueng, H. L. W. Chan, C. Surya, and C. L. Choy, Piezoelectric Coefficient of Aluminum Nitride and Gallium Nitride, J. Appl. Phys. 88, 5360 (2000).

[28] O. Ambacher, R. Dimitrov, M. Stutzmann, B. E. Foutz, M. J. Murphy, J. A. Smart, J. R. Shealy, N. G. Weimann, K. Chu, M. Chumbes et al., Two-Dimensional Electron Gases Induced by Spontaneous and Piezoelectric Polarization Charges in N- and Ga-Face AlGaN/GaN Heterostructures, Phys. Status Solidi (b) 216, 381 (1999).

[29] I. Vurgaftman and J. R. Meyer, Band Parameters for Nitrogen-Containing Semiconductors, J. Appl. Phys. 94, 3675 (2003).

[30] A. Hangleiter, F. Hitzel, S. Lahmann, and U. Rossow, Composition Dependence of Polarization Fields in GaInN/GaN Quantum Wells, Appl. Phys. Lett. 83, 1169 (2003).

[31] F. Renner, P. Kiesel, G. H. Döhler, M. Kneissl, C. G. Van de Walle, and N. M. Johnson, Quantitative Analysis of the Polarization Fields and Absorption Changes in InGaN/GaN Quantum Wells with Electroabsorption Spectroscopy, Appl. Phys. Lett. 81, 490 (2002).

[32] D. Turchinovich, P. Uhd Jepsen, B. S. Monozon, M. Koch, S. Lahmann, U. Rossow, and A. Hangleiter, Ultrafast Polarization Dynamics in Biased Quantum Wells under Strong Femtosecond Optical Excitation, Phys. Rev. B 68, 241307 (2003).

[33] H. Zhang, E. J. Miller, E. T. Yu, C. Poblenz, and J. S. Speck, Analysis of Interface Electronic Structure in $\mathrm{In}_{x} \mathrm{Ga}_{1-x} \mathrm{~N} / \mathrm{GaN}$ Heterostructures, J. Vac. Sci. Technol. B 22, 2169 (2004).

[34] D. Cherns, J. Barnard, and F. A. Ponce, Measurement of the Piezoelectric Field across Strained InGaN/GaN Layers by Electron Holography, Solid State Commun. 111, 281 (1999).

[35] A. S. Barker and M. Ilegems, Infrared Lattice Vibrations and Free-Electron Dispersion in GaN, Phys. Rev. B 7, 743 (1973).

[36] O. Madelung, Semiconductors: Data Handbook (Springer, Berlin-Heidelberg, 2004).

[37] Y.-R. Wu, R. Shivaraman, K.-C. Wang, and J. S. Speck, Analyzing the Physical Properties of InGaN Multiple Quantum Well Light Emitting Diodes from Nano Scale Structure, Appl. Phys. Lett. 101, 083505 (2012).

[38] P. M. McBride, Q. Yan, and C. G. Van de Walle, Effects of In Profile on Simulations of InGaN/GaN Multi-Quantum-Well Light-Emitting Diodes, Appl. Phys. Lett. 105, 083507 (2014). 
[39] N. Grandjean, B. Damilano, S. Dalmasso, M. Leroux, M. Laügt, and J. Massies, Built-in Electric-Field Effects in Wurtzite AlGaN/GaN Quantum Wells, J. Appl. Phys. 86, 3714 (1999).

[40] M. Leroux, N. Grandjean, J. Massies, B. Gil, P. Lefebvre, and P. Bigenwald, Barrier-Width Dependence of Group-III Nitrides Quantum-Well Transition Energies, Phys. Rev. B 60, 1496 (1999).

[41] E. T. Yu, G. J. Sullivan, P. M. Asbeck, C. D. Wang, D. Qiao, and S.S. Lau, Measurement of Piezoelectrically Induced Charge in GaN/AlGaN Heterostructure Field-Effect Transistors, Appl. Phys. Lett. 71, 2794 (1997).

[42] Y. Zhou, B. Shen, T. Someya, H. Yu, J. Liu, H. Zhou, R. Zhang, Y. Shi, Y. Zheng, and Y. Arakawa, Investigation of the Polarization-Induced Charges in Modulation-Doped $\mathrm{Al}_{x} \mathrm{Ga}_{1-x} \mathrm{~N} / \mathrm{GaN}$ Heterostructures through Capacitance Voltage Profiling and Simulation, Jpn. J. Appl. Phys. 41, 2531 (2002).

[43] K. Köhler, S. Müller, P. Waltereit, W. Pletschen, V. Polyakov, T. Lim, L. Kirste, H. P. Menner, P. Brückner, O. Ambacher, C. Buchheim, and R. Goldhahn, Electrical Properties of $\mathrm{Al}_{x} \mathrm{Ga}_{1-x} \mathrm{~N} / \mathrm{GaN}$ Heterostructures with Low Al Content, J. Appl. Phys. 109, 053705 (2011).

[44] S. K. Davidsson, M. Gurusinghe, T. G. Andersson, and $\mathrm{H}$. Zirath, The Influence of Composition and Unintentional
Doping on the Two-Dimensional Electron Gas Density in AlGaN/GaN Heterostructures, J. Electron. Mater. 33, 440 (2004).

[45] J. Simon, R. Langer, A. Barski, M. Zervos, and N. T. Pelekanos, Residual Doping Effects on the Amplitude of Polarization-Induced Electric Fields in GaN/AlGaN Quantum Wells, Phys. Status Solidi (a) 188, 867 (2001).

[46] S. Arulkumaran, T. Egawa, H. Ishikawa, and T. Jimbo, Characterization of Different Al Content $\mathrm{Al}_{x} \mathrm{Ga}_{1-x} \mathrm{~N}$ Heterostructures and High-Electron-Mobility Transistors on Sapphire, J. Vac. Sci. Technol. B 21, 888 (2003).

[47] K. A. Mkhoyan, J. Silcox, Z. Yu, W. J. Schaff, and L. F. Eastman, Formation of a Quasi-Two-Dimensional Electron Gas in $\mathrm{GaN} / \mathrm{Al}_{x} \mathrm{Ga}_{1-x} \mathrm{~N}$ Heterostructures with Diffuse Interfaces, J. Appl. Phys. 95, 1843 (2004).

[48] Y. Zhang, I. P. Smorchkova, C. R. Elsass, S. Keller, J. P. Ibbetson, S. P. DenBaars, U. K. Mishra, and J. Singh, Charge Control and Mobility in AlGaN/GaN Transistors: Experimental and Theoretical Studies, J. Appl. Phys. 87, 7981 (2000).

[49] W. Li, S. Sharmin, H. Ilatikhameneh, R. Rahman, Y. Lu, J. Wang, X. Yan, A. Seabaugh, G. Klimeck, D. Jena, and P. Fay, Polarization-Engineered III-Nitride Heterojunction Tunnel Field-Effect Transistors, IEEE J. Explor. Solid-State Comput. Devices Circuits 1, 28 (2015). 